\title{
Compromiso e influencia del equipo directivo desde la resiliencia socioeducativa
}

\section{Commitment and Influence of Socio-Educationally Resilient Administrative Teams}

Sonia Salvo-Garrido (*) https://orcid.org/0000-0002-9567-2285

Pilar Cisternas-Salcedo (*) https://orcid.org/0000-0003-0736-4547

(*) Universidad de la Frontera

(Recibido: 29 de marzo de 2019; Aceptado para su publicación: 19 de junio de 2019)

Cómo citar: Salvo-Garrido, S y Cisternas-Salcedo, P. (2021). Compromiso e influencia del equipo directivo desde la resiliencia socioeducativa. Revista Electrónica de Investigación Educativa, 23, e01, 1-16. https://doi.ora/10.24320/redie.2021.23.e01.2949

\section{Resumen}

En los procesos de selección del equipo directivo, el liderazgo es visto como una competencia individual. Debido a la actual transformación sociocultural, el liderazgo debe ser observado desde la perspectiva del capital social, donde lo colaborativo reemplaza a la administración burocrática. Este cambio de paradigma provoca conflictos internos relacionados con el compromiso e influencia que tienen tanto sobre la comunidad como con el proyecto educativo. El objetivo de esta investigación fue determinar el grado de compromiso e influencia que posee el equipo directivo de establecimientos educativos resilientes de Chile a través de metodología cualitativa, análisis de contenido y matriz de impacto. Los resultados conducen a que compromiso e influencia son determinantes en la creación de un perfil de selección de directivo, donde el liderazgo transformacional es idóneo para cargos donde el capital social es evidente y el liderazgo transaccional es parte de las características de los cargos técnicos.

Palabras clave: capital social, influencia social, liderazgo.

\section{Abstract}

In selection processes for administrative teams, leadership is seen as an individual competence. In light of the current sociocultural transformation, leadership should be observed from a perspective of social capital, in which collaboration takes the place of bureaucratic administration. This paradigm shift gives rise to internal conflicts relating to their commitment and influence on the community and the educational project. The objective of this study was to determine the degree of commitment and influence of administrative teams in resilient educational institutions in Chile using a qualitative methodology, content analysis, and an impact matrix. The results show that commitment and influence are determining factors in creating a profile for selecting administrative teams, in which transformational leadership is key for positions with clear social capital and transactional leadership is one feature of technical positions. 


\section{Introducción}

Conocer la evolución del liderazgo, como concepto organizacional, es el punto de partida para el desarrollo - desde la teoría- de nuevas propuestas orientadas a la selección de equipo directivo para la Educación, considerando que la función directiva requiere de competencias específicas relacionadas con la función a ejercer. Desde el punto de vista organizacional, el liderazgo es una de las habilidades gerenciales más solicitadas por los sistemas de selección de personal para cargos directivos, entendiéndose como la habilidad de ejercer influencia sobre una persona (o grupo de personas) para la consecución de objetivos, en este caso orientados hacia el proyecto educativo, definición que está ligada a la idea de autoridad.

En el sector de la Educación, la selección de directivos en Chile depende del sistema de Alta Dirección Pública (ADP), cuyo objetivo es dotar profesionales con las competencias necesarias para la dirección de establecimientos educacionales con dependencia estatal (Agencia de Calidad de la Educación [ACE], 2017), estableciendo en su mandato institucional la búsqueda de directivos con capacidades (individuales) de liderazgo, relacionadas al ejercicio administrativo y no enfocadas en la capacidad de motivación y en el desarrollo de un alto nivel de compromiso con el proyecto educativo, sin usar el ejercicio de prácticas coercitivas(Arar y Abu Nasra, 2019).

Рara esta investigación se considera el liderazgo desde la Teoría del Rango Total (Ordóñez et al., 2017) a través del Multifactorial Leadership Questionnaire (MLQ), el cual resume los estilos de liderazgo en nueve dimensiones (figura 1).

Figura 1. Estilos y dimensiones del liderazgo según la Teoría de Rango Total y propuesta de denominación de los estilos de liderazgo

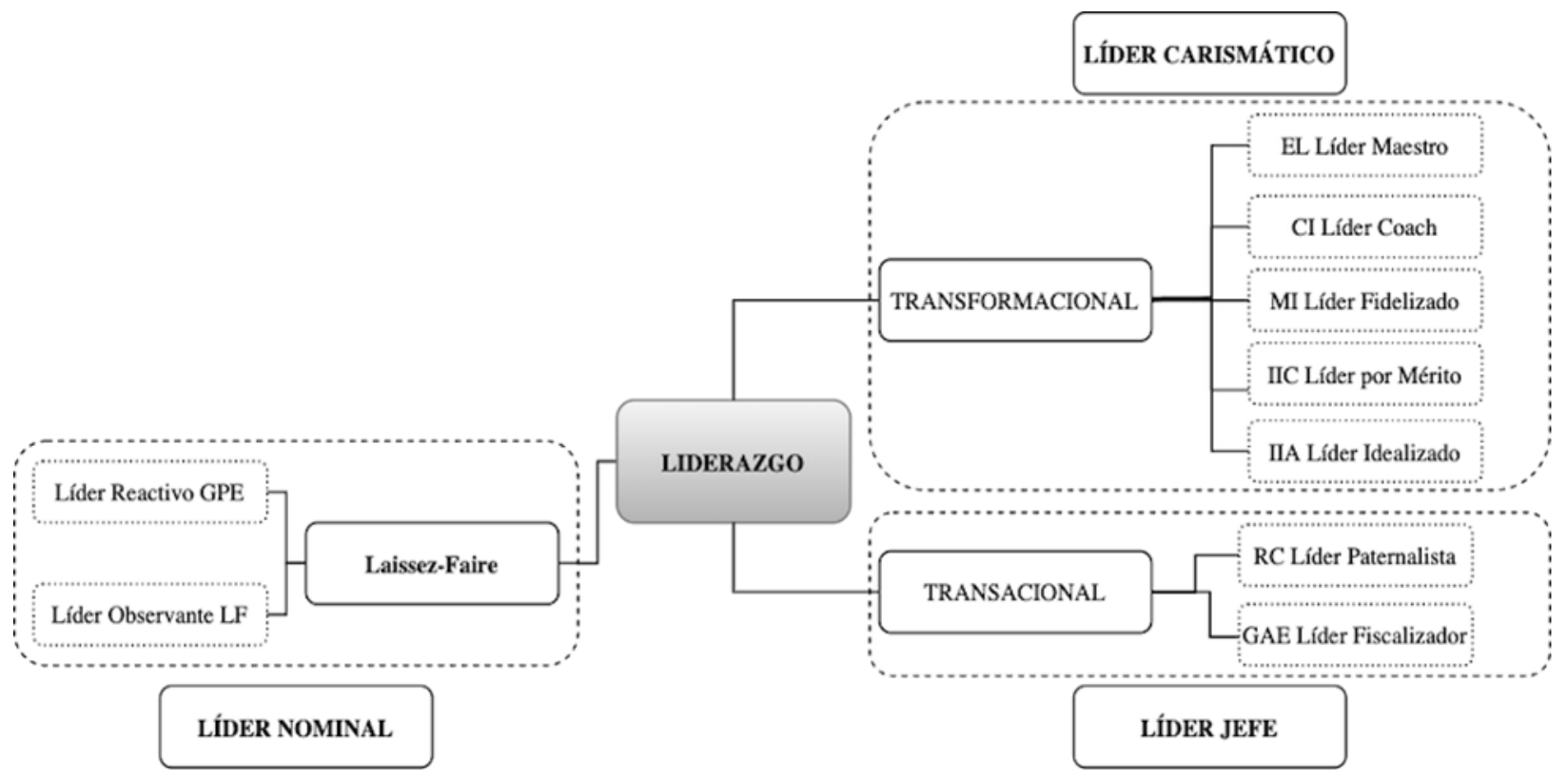

Liderazgo de Rango Total. La variedad conceptual del liderazgo, desde los múltiples enfoques y teorías, se resume en la capacidad de ejercer influencia sobre otro(s) (Weinstein, 2017) para la consecución de objetivos. Esta definición general muestra que la base del liderazgo es el ejercicio de la influencia sobre la autoridad (Davids y Waghid, 2019; Weinstein, 2017), donde ambos atributos son parte del poder, entendiendo la influencia como un proceso recíproco basado en la confianza y la autoridad como una demostración de poder desde lo burocrático e individual. 
La presente investigación se sustenta en el liderazgo desde la Teoría de Rango Total, propuesta por Bernard Bass desde la perspectiva de James Avolio (Garay, 2015; Ordóñez et al., 2017). Esta teoría divide al liderazgo en tres macroestilos, derivados de la Teoría de la Autoridad de Max Weber ${ }^{1}$ (Weber, 2005), los cuales son evaluados a través del MLQ (Bass y Avolio, 2014), validado en Chile por Vega y Zavala (2004), que define nueve dimensiones que caracterizan a los estilos de liderazgo en una organización desde la autopercepción (ver figura 1). Los tres macroestilos sugeridos por el Rango Total y las nueve dimensiones aportadas por el MLQ se describen a continuación:

Liderazgo Transaccional o de Desarrollo. Liderazgo ligado a la transacción, a la dualidad de la recompensa-castigo, como característica del "Jefe" en lo organizacional (Garay, 2015), manifestando una permanente negociación entre acciones constructivas y correctivas (Fernández y Quintero, 2017). Este tipo de líder se apega a la norma, con un sentido más individual y burocrático en el ejercicio del liderazgo. En este estilo, se presentan las siguientes dimensiones:

1. Recompensa Contingente, es un líder movido por la acción y la reacción, donde cada acierto es recompensado y cada falla es castigada. Aplica, en su ejercicio, modificadores de conducta. Рara efectos de este estudio se le denomina Líder Paternalista;

2. Gestión Activa por Excepción, este tipo de líder se caracteriza por estar atento a la efectividad del trabajo realizado, ejerciendo una constante supervisión y fiscalización en la búsqueda de hallazgos y no-conformidades. Para efectos de este estudio se le denomina Líder Fiscalizador.

Liderazgo Transformacional. Liderazgo relacionado con la habilidad de transformar el clima laboral a través de su capacidad de trabajo en equipo (Garay, 2015), al refuerzo positivo (Ordóñez et al., 2017) y a la adaptación a los cambios (Fernández y Quintero, 2017). Este estilo de liderazgo se caracteriza por el uso positivo del carisma, que contribuye a la proyección de una imagen de admiración y respeto. En este tipo de liderazgo se identifican las dimensiones:

1. Estimulación Intelectual: esta dimensión de liderazgo está asociada a la innovación y a la estimulación del pensamiento crítico. Para efectos de este estudio se le denomina Líder Maestro.

2. Consideración Individualizada: este tipo de líder se enfoca en las necesidades individuales, fomentando el desarrollo de potenciales y capacidades. Рara efectos de este estudio se le denomina Líder Coach.

3. Motivación Inspiracional: este líder está apegado al cumplimiento de la misión del proyecto organizacional, característica relacionada al sistema "empresa", por lo que para efectos de este estudio se le denomina Líder Fidelizado.

4. Influencia Idealizada Conductual: este tipo de liderazgo se hace evidente en aquellos funcionarios que han ejercido labores dentro de toda la escala jerárquica. Conocer debilidades y fortalezas de cada escalafón, lo convierte en un "modelo a seguir". Para efectos de este estudio se le denomina Líder por Mérito.

5. Influencia Idealizada Atribuida: esta dimensión basa su liderazgo en el poder del respeto y admiración, por lo que es idealizado por subalternos y pares. Para efectos de este estudio se le denomina Líder Idealizado.

Liderazgo Laissez-Faire/Pasivo/Correctivo/Evitador. Es un antagonista del liderazgo, que no asume responsabilidades, ya que "evitan tomar decisiones y verse implicados en los asuntos importantes" (Ordóñez et al., 2017, p. 5). Sus dimensiones son:

\footnotetext{
${ }^{1}$ Teoría propuesta a inicios del siglo XX, con edición póstuma en 1958: Die drei reinen Typen der legitimen Herrschaft, con reediciones posteriores.
} 
1. Gestión Pasiva por Excepción: su actuar sólo se evidencia ante normas no cumplidas; sólo ejerce liderazgo cuando nadie más quiere hacerlo. Para efectos de este estudio se le denomina Líder Reactivo.

2. Laissez-Faire: no ejerce liderazgo alguno; permanece ajeno a responsabilidades y toma de decisiones. Para efectos de este estudio se le denomina Líder Observante.

\subsection{Desde lo individual a lo colectivo: el rol del capital social}

De acuerdo con el sistema de selección ADP (ACE, 2017; Aravena y Quiroga, 2018), el liderazgo es considerado como atributo personal (Davids y Waghid, 2019), formando parte del perfil de competencias que debe poseer un directivo, cuya función está centrada en labores administrativas, financieras y pedagógicas (Bolivar y Murillo, 2017; Vázquez et al., 2016), dando lugar al desarrollo de un liderazgo burocrático basado en la jerarquía (Blossing y Liljenberg, 2018), división del trabajo y cumplimiento de normas (Lumby, 2019), sin considerar ni soluciones creativas (Li et al.; 2016) ni la adaptación al entorno sociocultural en el que se inserta el establecimiento educacional. De la misma manera, en el sistema educativo formador de profesionales de la Educación se considera al liderazgo como una de las competencias individuales intelectuales deseadas en el perfil del futuro profesor (Ugarte y Naval, 2010).

Esta mirada burocrática del liderazgo, con marcada influencia de Weber (2005), busca el ejercicio del mismo, desde una perspectiva individual y organizacional (Katewa y Heystek, 2019) esperando que este desarrollo de capacidades individuales sea necesariamente exitosa en el trabajo colaborativo (Álvarez et al., 2016). De esta manera, desde una perspectiva burocrática, el rol de líder directivo queda reducido sólo a la función administrativa de carácter gerencial (Katewa y Heystek, 2019; Blossing y Liljenberg, 2018; Heck y Hallinger, 2010), donde existe una falsa percepción de liderazgo, el cual correspondería más bien al ejercicio unilateral de la autoridad (Davids y Waghid, 2019; Weinstein, 2017).

La propuesta de Borrero y Gamboa (2016) relacionada con la función del liderazgo como nexo entre la gestión administrativa y las relaciones sociales (figura 2) dentro del sistema directivo escolar, corresponde a la labor ejercida para balancear la gestión pedagógico-administrativa y la Comunidad Educativa (Li et al., 2016), conformada por docentes, funcionarios y alumnos (figura 3), coincidiendo con el carácter burocrático propuesto por Weber, donde se refuerza el mandato a través de la jerarquía y cumplimiento de la norma.

Figura 2. Función del liderazgo en la organización

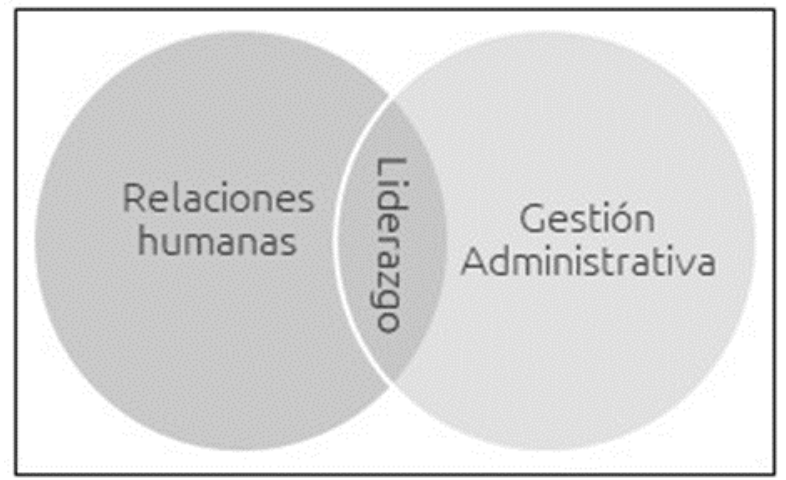

Fuente: Elaboración propia a partir de Borrero y Gamboa (2016). 
Figura 3. Función del liderazgo en la organización

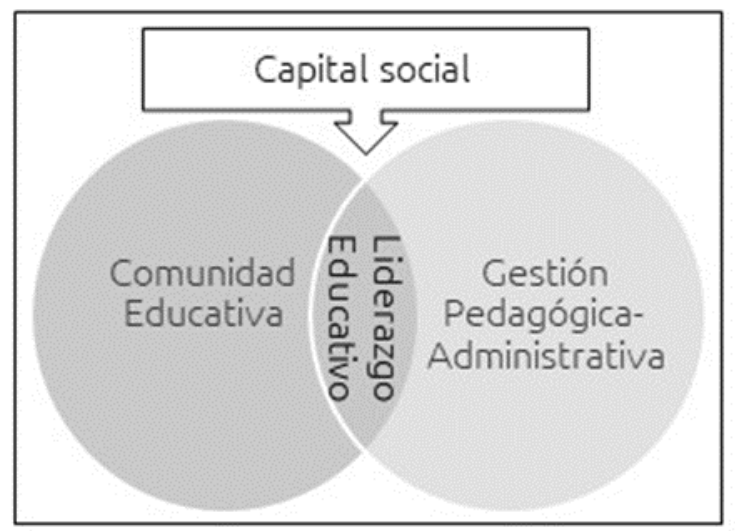

Fuente: Elaboración propia a partir de Borrero y Gamboa (2016).

El liderazgo, como propuesta para las buenas prácticas directivas en Educación, debe ser considerado como un proceso social (Redondo y Elboj, 2018), desde una perspectiva colectiva más que como atributo personal, donde es el capital social el potenciador de este aspecto colectivo, a través de la generación de redes, lo que contribuye a disminuir el "aislamiento profesional" (Hernández y Navarro, 2018, p. 35), en pos del cuestionamiento sobre la rigidez estructural del sistema educativo tradicional, contribuyendo al desarrollo de nuevas metodologías de enseñanza. De esta manera, el liderazgo debe ser conceptualizado en términos de relaciones sociales, donde la confianza (como factor relacional del capital social) promueve el desarrollo de la motivación y el compromiso hacia el proyecto educativo(Davids y Waghid, 2019).

Desde las buenas prácticas, el liderazgo directivo educativo conlleva un compromiso ético donde el mejoramiento de la calidad es integral, abarcando tanto el aprendizaje del alumnado (y desarrollo de la comunidad educativa en pleno) como la capacitación de docentes y funcionarios (Hernández-Castilla et al., 2017; Vicente de Vera y Gabari, 2019). Desde esta perspectiva es donde la aseveración de Borrero y Gamboa (2016) debe ser ampliada hacia un liderazgo con características colectivas y territoriales que sólo el capital social le puede proporcionar (ver figura 4).

Figura 4. Función del liderazgo directivo educativo desde el capital social

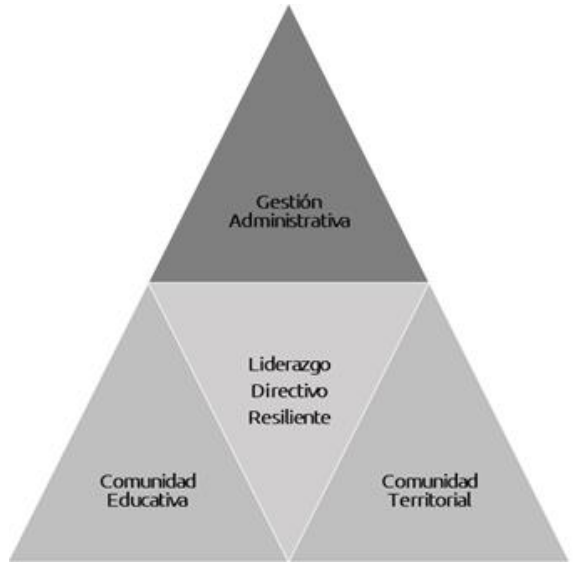

El carácter colectivo del capital social influye en el liderazgo a través de prácticas transformacionales, pero surgen las siguientes interrogantes: ¿será suficiente el liderazgo transformacional, como atributo, dentro del equipo directivo?, ¿el liderazgo transformacional garantiza el compromiso y la influencia sobre el proyecto educativo? La presente investigación trata de dar una perspectiva desde la Teoría de Rango Total, 
el MLQ y el capital social, para la caracterización de los tipos de liderazgo necesarios en el equipo directivo, como base para la selección de personal.

\subsection{Compromiso desde el capital social}

Si desde la perspectiva de la selección de personal se considera al liderazgo como un atributo personal, el capital social proporciona el atributo colectivo, donde el recurso invertido en redes sociales es responsabilidad de los mismos integrantes de la red; por lo tanto, el carácter burocrático e individual del liderazgo tradicional se convierte en un liderazgo en lo colectivo, dejando de "responder a las características individuales" (Redondo y Elboj, 2018, p. 110) para definir al liderazgo como proceso social, donde el carácter multidimensional del capital social refuerza la estructura del liderazgo en la educación (figura 5).

Figura 5. Multidensionalidad del capital social y relaciones entre nodos

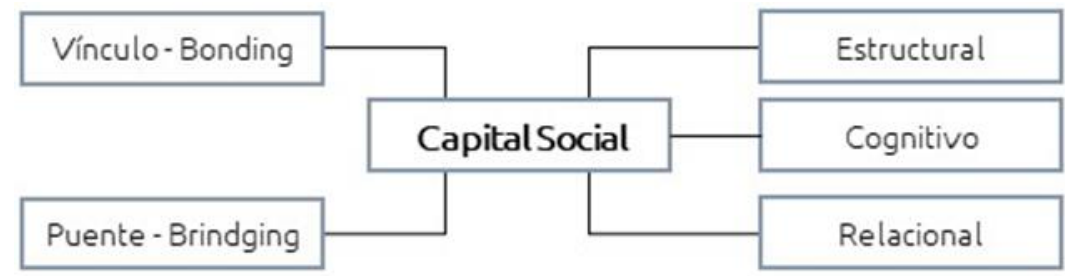

Desde la teoría, el capital social se refiere a la formación de redes y a la confianza que se genera dentro y entre ellas (Lin et al., 2008), como una forma de capturar el trabajo colaborativo (Osmond-Johnson, 2018) dentro de una comunidad y desde el valor público, donde el individuo se beneficia de la comunidad y la comunidad se beneficia del capital social generado por la interacción de los individuos (Diep et al., 2017; Osmond-Johnson, 2018). Este beneficio recíproco deja entrever el carácter colaborativo de la influencia que se ejerce desde el liderazgo (Weinstein, 2017).

Dentro de la organización, el capital social fluctúa entre el vínculo/bonding, que son los recursos en la forma de lazos entre pares (Neves et al., 2019), y el puente/bridging, donde los recursos radican en el establecimiento de canales de comunicación dentro de la jerarquía organizativa. La intensidad del vínculo y del puente depende de muchos factores, pero principalmente los que están ligados a la vulnerabilidad social (Neves et al., 2019), donde la resiliencia debe actuar desde el capital social a través de la generación de confianza, refuerzo positivo y del anhelo de conseguir metas (Trujillo et al., 2011). Bajo esta visión del capital social, el compromiso con el proyecto educativo se da en el refuerzo continuo de las relaciones del tipo vincular, dentro del estamento directo; mientras que las relaciones tipo puente refuerzan el concepto de influencia desde la plana directiva hacia la comunidad educativa.

Desde la multidimensionalidad del capital social, el compromiso organizacional es visto como el nivel de implicancia de los individuos en un determinado proyecto, donde la influencia ejerce un efecto colaborativo desde el carácter relacional del capital social. Este juego entre la influencia y el compromiso se desarrolla desde el capital social, donde la influencia recibe la apuesta desde lo relacional (confianza) y el compromiso desde lo cognitivo (proyecto educativo).

El concepto de capital social, para esta investigación, estará asociado al enfoque multidimensional propuesto por Zheng (2010), que contempla las dimensiones estructural (comunidad educativa y comunidad territorial), cognitiva (proyecto educativo) y relacional (confianza) (Delgado et al., 2011; López et al., 2018).

\subsection{Liderazgo directivo desde la resiliencia}

El concepto original de resiliencia estaba orientado al proceso de adaptación a experiencias negativas (Yee y Sulaiman, 2017). Este proceso reúne aspectos ligados a la psicología y el comportamiento, por lo que la 
resiliencia es un proceso dinámico y progresivo, en el que se involucran factores de riesgo y factores protectores (Tansey et al., 2017), tal como lo propuso Kumpfer (1999) en su modelo de resiliencia, el cual señala la importancia de los factores internos, situacionales y contexto socioambiental como elementos protectores o de riesgo (Tansey et al., 2017), constructos necesarios para la predicción de comportamientos resilientes (Kumpfer, 1999) tanto del alumno como del docente y cuerpo directivo. En el caso del personal directivo, los factores de riesgo y protección siguen la dinámica propuesta por Kumpfer; la diferencia con la resiliencia socioeducativa reside en el "nivel de experiencia, estilo atribucional, personalidad, etc." (Vicente de Vera y Gabari, 2018, p. 108), características que son adquiridas a través de los años de ejercicio de la carrera docente.

El papel del liderazgo en los establecimientos educativos es uno de los factores más relevantes en la calidad del aprendizaje (Leithwood, 2009; Weinstein, 2017), que cobra mayor relevancia en ambientes de vulnerabilidad social. Al incorporar los elementos del capital social, el liderazgo adquiere características comunitarias, donde el entorno en el que se encuentra inserto el establecimiento educativo juega un rol decisivo en la forma de ejercer el liderazgo, donde la vulnerabilidad social actúa como factor de riesgo en el desarrollo socioeducativo de los alumnos, apareciendo la resiliencia como la capacidad positiva de recuperarse a emociones negativas y a la adaptación necesaria para iniciar esta recuperación, y en el caso de la resiliencia socioeducativa, la recuperación se realiza dentro de ese contexto de vulnerabilidad social.

En la resiliencia, algunos de los factores protectores son la autoestima y la generación de redes, y como reductor del impacto de los factores de riesgo está la formación de vínculos entre pares, siendo la autoestima, la generación de redes y la formación de vínculos entre pares las características de la multidimensionalidad del capital social.

La efectividad del líder está en el constante interés por el desarrollo de competencias (Somprach y Ngang, 2018), en su capacidad de adaptación a las circunstancias (Vázquez et al., 2016) y en la consideración por los aspectos culturales (Rooney, 2018), por lo que la resiliencia no es un concepto ajeno a su labor, ya que el directivo escolar está desarrollando de manera permanente el rol de consejero y defensor (OsmondJohnson, 2018) como parte de su batería de habilidades blandas.

De acuerdo con la propuesta de Borrero y Gamboa (2016), el líder directivo actúa como nexo entre la Administración y la Comunidad Educativa; en el caso de la resiliencia, el líder directivo ingresa al esquema a través de ella, utilizando las características del capital social para llevar a cabo el proyecto educativo, a modo de una constante superación de obstáculos, donde el líder directivo, a través de sus propias habilidades blandas (como las sugeridas por Osmond-Johnson, 2018) surge como líder resiliente (figura 6).

Figura 6. Liderazgo directivo como potenciador de la resiliencia educativa

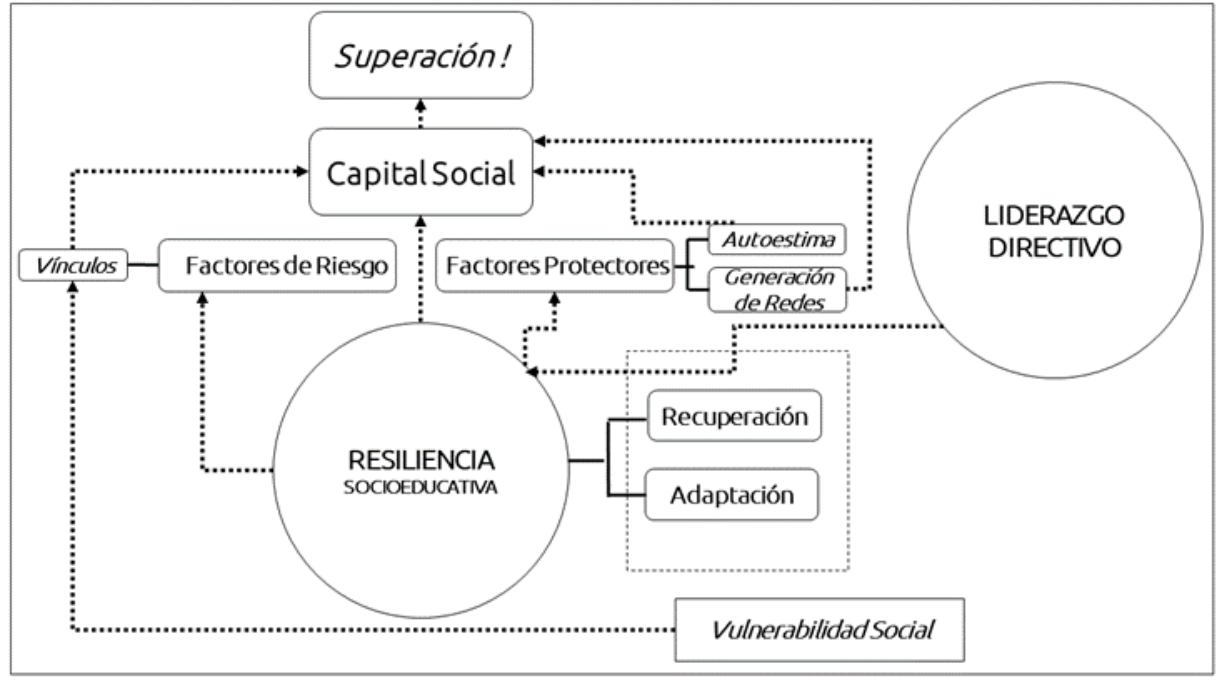


Es así como el líder directivo resiliente utiliza la creación de confianza del capital social como instrumento de mediación para la ejecución del proyecto educativo a través de la capacidad de generar motivación y compromiso por parte del plantel docente y el alumnado (Arar y Abu Nasra, 2019; Li et al., 2016).

Por lo expuesto antes, esta investigación tiene como objetivo determinar el grado de compromiso del equipo directivo y su grado de influencia sobre el proyecto educativo de los establecimientos educacionales resilientes de Chile.

\section{Método}

Para el estudio se utilizó un diseño metodológico de carácter cualitativo-transversal, a través del análisis de contenido, el cual permite analizar la naturaleza del discurso (Porta y Silva, 2003), asignando a cada unidad de análisis un código de manera inductiva y deductiva (González-Teruel, 2015), comparando y categorizando datos dentro del texto (Gerbic y Stacey, 2005). Además se utilizó el método MACTOR (Matrix of Alliances and Conflicts: Tactics, Objectives and Recommendations) para determinar las relaciones de influencia y compromiso entre los actores relevantes (Popp et al., 2018), dados por las dimensiones del liderazgo según el MLQ (Bass y Avolio, 2014; Mendoza et al., 2014; Ordóñez et al., 2017; Vega y Zavala, 2004).

Se realizó una selección de 24 profesionales, miembros del estamento directivo (Director, Jefe Unidad Técnico-Pedagógica, Orientador) de establecimientos educacionales con características de vulnerabilidad social, en donde existían alumnos con trayectoria resiliente. Dichos establecimientos educacionales están ubicados en las regiones de Arica-Parinacota, Antofagasta, Metropolitana, Bío Bío y Ñuble.

Se aplicaron 24 entrevistas semiestructuradas basadas en las preguntas del Cuestionario Multifactorial de Liderazgo propuesto por Bass y Avolio (2014) y Mendoza et al. (2014) en su versión corta MLQ-5x (Ordóñez et al., 2017), validado en Chile por Vega y Zavala (2004), el cual mide la autopercepción de liderazgo y su respectiva identificación dimensional. Se utilizó como base de la investigación el MLQ-5x debido a la confiabilidad de los resultados (Báez et al., 2019). La edad de los profesionales varió entre 37 y 61 años (Media = 50.3 y Desviación estándar = 7.6) y la mayoría fueron mujeres (53.8\%).

Las nueve dimensiones teóricas propuestas por el MLQ-5x (ver figura 1) fueron incluidas en las entrevistas como preguntas que generaron "directrices sistemáticas, pero flexibles para la recolección y análisis de datos" (Charmaz en González Teruel, 2015, p. 323).

El análisis de datos se realizó en dos fases: la primera, mediante análisis de contenido a través del software Atlas.ti 7.5.18 para la obtención de códigos para las variables y dimensiones sugeridas por Bass y Avolio, (2014); Bass et al. (2000); Vega y Zavala (2004); la segunda fase fue de análisis de actores a través de matriz de impacto según el modelo MACTOR, metodología basada en la Teoría de Juegos (Rico, 2018), que busca valorar las relaciones de fuerza entre los actores y estudiar sus convergencias y divergencias con respecto a un cierto número de posturas y de objetivos asociados (Godet y Durance, 2011). Este método facilita la decisión de la puesta en marcha de su política de alianzas y de conflictos al definir la posición de cada uno de los actores (en este caso liderazgos) frente a los objetivos (o misión del proyecto educativo) (Belfellah y Gassemi, 2016). Esta metodología facilita los procesos de articulación y el mejoramiento de políticas y procesos institucionales al tomar en cuenta a los actores (las dimensiones de la Teoría de Rango Total, como individualización de los liderazgos) que tienen interés en las reformas o propuestas y que están, o podrían estar, involucrados en el proyecto.

\section{Resultados}

Según la metodología MACTOR de matriz de impacto, las nueve dimensiones de los estilos de liderazgo propuestas por la Teoría del Rango Total (ver figura 1) se distribuyen en la matriz formando clústeres, donde cada cuadrante indica grado de influencia (Godet y Durance, 2011) que ejerce el liderazgo sobre el proyecto educativo. Tal como se puede observar en la figura 7, en el cuadrante superior izquierdo se posiciona el Líder Maestro junto al Líder Coach, esta posición es de dominancia, donde ambos tipos de 
liderazgo son los que mayor influencia ejercen sobre la misión del proyecto educativo, mostrando que el liderazgo transformacional relacionado al refuerzo positivo, al desarrollo del potencial individual y a la estimulación del pensamiento crítico promueven el movimiento del capital social desde la autoconfianza, coincidiendo con uno de los factores de protección de la resiliencia socioeducativa.

En el cuadrante superior derecho se posicionan dos grupos: el primero incluye al Líder Paternalista junto al Líder Fiscalizador y el segundo incluye al Líder Observante junto al Líder Reactivo. Este cuadrante está relacionado con la función de apoyo, donde el cluster Paternalista-Fiscalizador es de características transaccionales y no tiene la influencia necesaria para determinar el rumbo del proyecto educativo, pero sí son fundamentales para el desarrollo de éste desde el punto de vista técnico-administrativo. De la misma forma, el clúster Observante-Reactivo, correspondiente al liderazgo (o antiliderazgo) del tipo Laissez-Faire; al posicionarse en el mismo cuadrante indica que la actitud pasiva y reactiva del laissez-faire no es determinante en el fin último que es la misión del proyecto educativo, pero sí puede influir a través de la falta de interés en la toma de decisiones desde la arista administrativa.

En el cuadrante inferior derecho, relacionado con atender las decisiones, se posiciona el clúster formado por el Líder por Mérito, Líder Idealizado y Líder Fidelizado, los cuales presentan características transformacionales; esta posición le permite al liderazgo directivo moverse dentro del capital social a través de la generación de redes y el refuerzo de lazos (como vínculo y puente) y se relaciona con la resiliencia como factor protector y minimizador de los factores de riesgo. A pesar de que no tiene la influencia necesaria para determinar el rumbo del proyecto educativo, sí es relevante para actuar como promotor del trabajo colaborativo.

En el cuadrante inferior izquierdo se posicionan aquellos liderazgos autónomos que participan en la formulación del proyecto, pero no participan en la ejecución del mismo. En este cuadrante se ubicarían los responsables de diseñar políticas públicas locales y estatales, así como el cuerpo corporativo de aquellos establecimientos educacionales pertenecientes a Redes Educativas, que en esta investigación no fueron considerados.

Figura 7. Influencia de las dimensiones de liderazgo en el cumplimiento del proyecto educativo

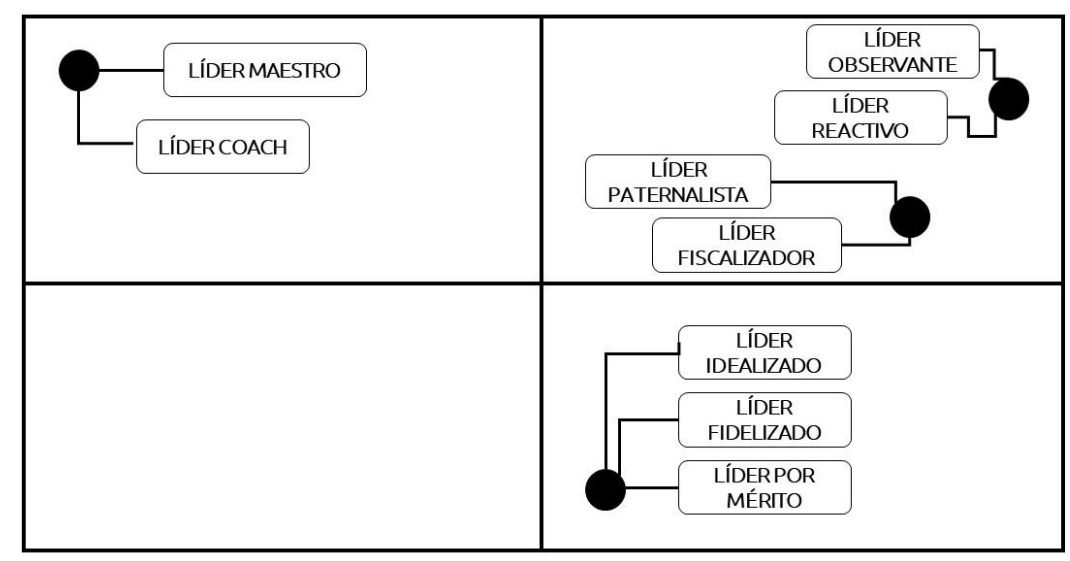

La siguiente matriz de impacto (figura 8) evidencia el grado de compromiso por parte de los distintos tipos de liderazgo con el proyecto educativo, donde la lejanía de los nodos muestra un compromiso menos arraigado o un menor convencimiento de la misión del proyecto. 
Figura 8. Compromiso de las dimensiones de liderazgo con el proyecto educativo

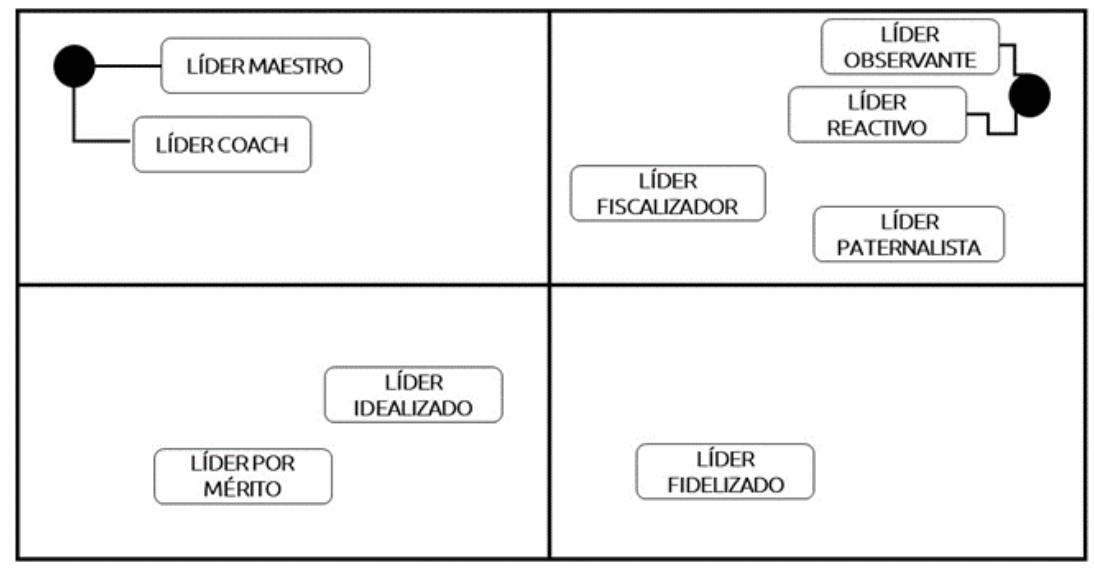

El cuadrante superior izquierdo (al igual que en la figura 7) indica dominancia. En esta posición se aprecia que el clúster Líder Maestro-Líder Coach es el más comprometido con el proyecto educativo, demostrando que el liderazgo transformacional -a través del refuerzo positivo, desarrollo del potencial individual y la estimulación del pensamiento crítico- actúa como gestor y potenciador del capital social, desde la confianza, hacia la "apropiación" (en el sentido del "compromiso con convencimiento"; por ende, hacerlo suyo) del proyecto educativo.

En el cuadrante superior derecho se ubican los "actores de reparto", aquellos que ejercen una labor de apoyo al proyecto. En esta posición está el Líder Fiscalizador y el Líder Paternalista, cada uno actuando de manera independiente, sin formar una alianza o clúster. Ambos Líderes desarrollan un rol de apoyo al proyecto educativo desde el liderazgo transaccional (ver figura 1): el Líder Fiscalizador apoya desde su visión técnico-administrativa, en un rol de supervisión del cumplimiento administrativo del proyecto educativo, mientras que el Líder Paternalista, dentro de la misma función de apoyo, aporta desde la supervisión del recurso humano que participa en el proyecto educativo. Por lo tanto, el grado de compromiso de ambos líderes es menor, porque su función está determinada por características individuales de lo transaccional, ya que el objetivo de ambos líderes es el cumplimiento del mandato. En este mismo cuadrante se ubica el clúster formado por el Líder Reactivo y el Líder Observante, ambos pertenecientes al estilo Laissez-Faire. En este caso, el carácter antagonista de ambos liderazgos debe ser visto como un anti-apoyo al proyecto educativo. Este clúster posee un grado de compromiso forzado, un compromiso que no se hace presente si no es solicitado.

En el cuadrante inferior derecho, que se relaciona con una posición alerta, se ubica el Líder Fidelizado. Este liderazgo asume un tipo de compromiso condicionado a la misión del proyecto educativo desde la perspectiva del trabajo colaborativo: el apoyo será total, siempre y cuando la comunidad educativa se comprometa.

En el cuadrante inferior izquierdo se ubican el Líder por Mérito y el Líder Idealizado, ambos con características autónomas. En el caso de estos liderazgos, el compromiso con el proyecto educativo no es tan arraigado, está orientado al compromiso con la comunidad educativa denotando el carácter transformacional -más ligado a la creación de confianza que el clúster Líder Maestro-Líder Coach.

\section{Discusión}

El liderazgo en la educación se ha enfrentado a cambios que van desde lo instrumental a lo estructural. El liderazgo, desde lo organizacional, fue introducido en la educación a través de los procesos de selección, cuyas directrices estaban enfocadas en la individualidad del cargo. El liderazgo de hoy representa el dejar de lado el concepto de Director Escolar (Álvarez et al., 2016) y reemplazarlo por Líder Educativo Resiliente, 
quien resume en su labor la intensidad del capital social a través de la creación de confianza, generación de redes y compromiso con el proyecto educativo.

Desde la Teoría del Rango Total en su estado puro, el liderazgo es concebido como una práctica ligada al concepto de burocracia propuesta por Weber (2005). Al incorporar las características del capital social, el liderazgo adquiere el poder necesario (como compromiso e influencia) para acoplar a la Comunidad Territorial (redes sociales que están fuera de la Comunidad Educativa y entorno socioeconómico donde se inserta la escuela) al sistema propuesto por Borrero y Gamboa (2016), referente al rol de liderazgo como nexo. Esta Comunidad Territorial, desde el punto de vista socioeconómico, debe ser integrada a través de la resiliencia, donde el capital social, una vez más, aporta la confianza (desde el compromiso) y la generación de redes (desde la influencia), transformando al directivo escolar en un líder directivo resiliente con la habilidad de generar cambios en un sistema mediante la creación y utilización de las redes sociales (Gurr, 2018), con la sabiduría, compromiso e influencia que adquiere a través de los años de ejercicio de la carrera docente (Vicente de Vera y Gabari, 2019).

El grado de influencia ejercido por el líder sobre el proyecto educativo y el grado de compromiso adquirido con el proyecto, se relacionan con el estilo de liderazgo:

- Liderazgo transformacional, con cualidades orientadas a la generación de confianza y redes (con más carga de capital social), posee más influencia sobre las decisiones que marcan la evolución del proyecto educativo. Es así como el clúster Líder Maestro-Coach posee más influencia sobre el destino del proyecto y mayor compromiso con el mismo, al intervenir directamente sobre el individuo, mediante el refuerzo positivo, desarrollo del potencial individual y estimulación del pensamiento crítico, coincidiendo con la base del capital social con respecto a la creación de confianza, y también con uno de los factores protectores de la resiliencia: la autoconfianza.

- Liderazgo transaccional, con cualidades orientadas al cumplimiento de la norma (con menor carga de capital social), posee una influencia moderada a baja sobre el proyecto educativo. En este caso, el clúster Líder Paternalista-Fiscalizador no tiene la capacidad de influir directamente, pero puede cambiar el destino de la misión del proyecto a través de su capacidad técnico-administrativa. En cuanto al grado de compromiso, es de carácter moderado con el proyecto educativo. Su intervención va por el lado del cumplimiento de la norma (Fiscalizador) y el control del recurso humano (Paternalista).

- Liderazgo Laissez-Faire, sin cualidades de liderazgo, con una orientación hacia el dejar ser (con carga nula de capital social). El clúster Líder Observante-Reactivo, a través de su indiferencia, tiene la capacidad de influir en el desarrollo del proyecto sobre el aspecto administrativo al no asumir responsabilidades. Con respecto al compromiso, no lo adquieren si no se les solicita.

Una situación curiosa ocurre con los líderes de la dimensión de la Influencia Idealizada (Atribuida e Idealizada), pertenecientes al estilo transformacional. Con respecto al grado de compromiso, ambos liderazgos poseen el atributo de la confianza del capital social desde el recurso humano (pares y subordinados), por lo que su compromiso y fidelización no está con la misión del proyecto educativo, si no con las personas que conforman el proyecto. Al ser autónomos, su utilidad es evidente para lograr cohesión en el equipo desde la vereda de la admiración y reconocimiento. De igual forma, la influencia ejercida por estos liderazgos es determinante si actúan como promotores del trabajo colaborativo.

Esta investigación sugiere que los liderazgos relacionados con el desarrollo de la autoconfianza son los que más influencia ejercen sobre el proyecto educativo, desde la perspectiva del capital social relacional, asimismo estos tipos de liderazgo son los que más se comprometen con la misión del proyecto. Los liderazgos que promueven el trabajo en red participan colaborando en el éxito de la misión del proyecto, a través del capital social estructural, donde el compromiso emana desde la comunidad educativa. De forma paralela, los liderazgos transaccionales influyen desde la promoción del proyecto educativo a través de la estructura administrativa, donde su compromiso en el éxito del proyecto educativo radica en el cumplimiento de la norma. 
De acuerdo con el análisis de impacto realizado en esta investigación, el estamento directivo debe contar con un equipo transformacional-transaccional (figura 9), en el que el proyecto educativo debe ser encabezado por un Director con características de Líder Coach-Maestro que promueve la generación de confianza, aportando el capital social relacional; un Jefe de Unidad Técnico-Pedagógica (encargado de organizar, coordinar y supervisar el trabajo técnico-pedagógico) con características de Líder Fiscalizador, que esté pendiente del cumplimiento de la norma desde el punto de vista organizacional, aportando el capital social cognitivo; y un Orientador (encargado de diagnosticar y atender necesidades de los alumnos y docentes) con características de Líder Fidelizado, que promueva el trabajo colaborativo desde el capital social estructural.

Figura 9. Propuesta de pauta de selección de personal directivo según estilos de liderazgo, grado de compromiso con la propuesta educativa y grado de influencia sobre el proyecto educativo

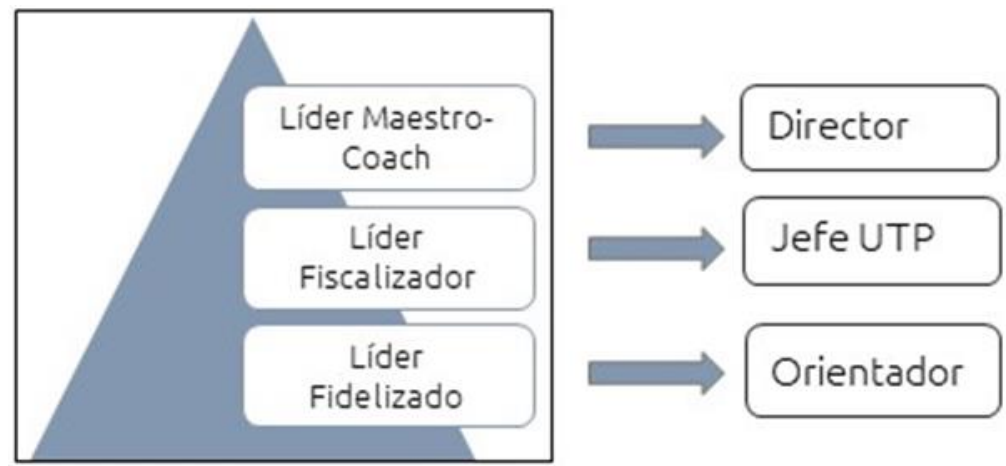

Según la idea de Borrero y Gamboa, el liderazgo es el nexo de las relaciones humanas y la administración. Este estudio demuestra que el liderazgo va mucho más allá de ser un nexo, es un continuo trabajar en habilidades blandas y en desarrollo personal para tomar las características del capital social a través de la resiliencia y formar un líder resiliente para el sector educativo.

El reconocimiento de la influencia del capital social en los estilos de liderazgo permitiría realizar una selección más acotada del personal idóneo para conformar el equipo directivo en los establecimientos educacionales. Esta investigación desnuda la situación del liderazgo directivo, sugiriendo que, hasta el momento, se enfatiza en el carácter individual (como atributo) y burocrático (en el ejercicio). Incorporando el capital social se transforma en liderazgo resiliente, en liderazgo colaborativo para el cuerpo docente, para el alumnado, para la familia y el entorno territorial de la escuela. Este liderazgo directivo resiliente muestra, a través de la dimensionalidad del MLQ, los grados de compromiso e influencia para con el proyecto educativo, lo que podría dar pie a la reformulación de políticas públicas orientadas a facilitar la selección de personal a través del sistema ADP.

\section{Agradecimientos}

Este artículo reporta resultados parciales del proyecto FONDECYT N¹160429, denominado "Factores protectores y de riesgo asociados a la resiliencia socioeducativa en estudiantes vulnerables de cuarto a octavo año de enseñanza básica: un modelo multidimensional explicativo de las trayectorias de evolución del rendimiento escolar en Chile", financiado por CONICYT y desarrollado entre marzo de 2016 y marzo de 2020.

\section{Referencias}

Agencia de Calidad de la Educación. (2017). Estudio Directores municipales seleccionados mediante la ley N. ${ }^{\circ} 20501$. Santiago de Chile.

http://archivos.agenciaeducacion.cl/Estudio directores municipales ley20501.pdf 
Álvarez, J., Torres, A. y Chaparro, E. (2016). Diagnóstico del liderazgo educativo en las Instituciones de Educación Superior del Valle de Toluca. Revista de Investigación Educativa, 34(1), 51-68.

https://doi.org/10.6018/rie.34.1.206881

Arar, K. y Abu Nasra, M. (2019). Leadership style, occupational perception and organizational citizenship behavior in the Arab education system in Israel. Journal of Educational Administration, 57(1), 101-116.

https://doi.org/10.1108/JEA-08-2017-0094

Aravena, F. y Quiroga, M. (2018). Autoetnografía y directivos docentes: una aproximación experiencial a las reformas educativas en Chile. Revista Electrónica de Investigación Educativa, 20(2), 113-125.

https://doi.org/10.24320/redie.2018.20.2.1600

Báez, F., Zurita, F., Martinez, A. y Zagalaz, M. L. (2019). Análisis psicométrico y relaciones de diagnóstico de la inteligencia emocional y liderazgo en docentes de enseñanzas regladas. Revista de Investigación Educativa, 37(1), 201-216. https://doi.org/10.6018/rie.37.1.308801

Bass, B. y Avolio, B. (2014). Multifactorial Leadership Questionnaire (MLQ). MindGarden.

Bass, B., Avolio, B. y Bass, B. (2000). MLQ Multifactor Leadership Questionnaire (5x-Short). https://www.mindgarden.com/content/27-researcher-established

Belfellah, Y. y Gassemi, K. (2016). Stratégies d'acteurs et démarche prospective?: Essai de formalisation des mécanismes de pouvoir au sein du secteur touristique marocain. [¿Estrategias de los actores y enfoque prospectivo?: intento de formalizar los mecanismos de poder dentro del sector turístico marroqui]. Recherches en Sciences de Gestion, 5(116), 25-49. https://doi.org/10.3917/resg.116.0025

Blossing, U. y Liljenberg, M. (2018). School leaders' relational and management work orientation. International Journal of Educational Management, 33(2), 276-286. https://doi.org/10.1108/ijem-07-2017$\underline{0185}$

Bolivar, A. y Murillo, F. J. (2017). El efecto escuela: un reto de liderazgo para el aprendizaje y la equidad. En J. Weinstein y G. Muñoz (Eds.), Mejoramiento y Liderazgo en la Escuela. Once Miradas (pp. 71-112). Centro de Desarrollo del Liderazgo Educativo.

Borrero, R. y Gamboa, M. (2016). La dirección de la gestión didáctica en la disciplina principal integradora de las carreras pedagógicas. Revista Didasc@lia: Didáctica y Educación, 7(5), 13-32.

Davids, N. y Waghid, Y. (2019). Educational leadership reconsidered: re-invoking authority in schools. Africa Education Review, 16, 1-14. https://doi.org/10.1080/18146627.2016.1257919

Delgado, M., Martín, G., Navas, J. E., Cruz, J., Navas, E. y Cruz, J. (2011). Capital social, capital relacional e innovación tecnológica. Una aplicación al sector manufacturero español de alta y media-alta tecnología. Cuadernos de Economía y Dirección de la Empresa, 14(4), 207-221.

Diep, N. A., Cocquyt, C., Zhu, C., Vanwing, T. y de Greef, M. (2017). Effects of core self-evaluation and online interaction quality on adults' learning performance and bonding and bridging social capital. Internet and Higher Education, 34, 41-55. https://doi.org/10.1016/i.iheduc.2017.05.002

Fernández, C. y Quintero, N. (2017). Liderazgo transformacional y transaccional en emprendedores venezolanos. Revista Venezolana de Gerencia, 22(77), 56-74.

Garay, S. (2015). Liderazgo y logros en las organizaciones escolares de Chile (Tesis Doctoral). Universidad Complutense. 
Gerbic, P. y Stacey, E. (2005). A purposive approach to content analysis: designing analytical frameworks. The Internet and Higher Education, 8(1), 45-59.

Godet, M. y Durance, P. (2011). La prospective stratégique por les enterprises et les territoires. Dunod. https://doi.org/10.3917/dunod.godet.2011.01

González-Teruel, A. (2015). Estrategias metodológicas para la investigación del usuario en los medios sociales: análisis de contenido, teoría fundamentada y análisis del discurso. El Profesional de la Información, 24(3), 321-328. https://doi.org/10.3145/epi.2015.may.12

Gurr, D. (2018). School middle leaders in Australia, Chile and Singapore. School Leadership \& Management, 1-19. https://doi.org/10.1080/13632434.2018.1512485

Heck, R. H. y Hallinger, P. (2010). Testing a longitudinal model of distributed leadership effects on school improvement. Leadership Quarterly, 21(5), 867-885. https://doi.org/10.1016/j.leaqua.2010.07.013

Hernández, E. y Navarro, M. J. (2018). Redes educativas locales para la mejora escolar. Revista Electrónica de Investigación Educativa, 20(3), 29-42. https://doi.org/10.24320/redie.2018.20.3.1669

Hernández-Castilla, R., Murillo, F. J. y Hidalgo, N. (2017). Lecciones aprendidas del estudio del liderazgo escolar exitoso. Los casos de España en el Proyecto Internacional ISSPP. Revista de Investigacion Educativa, 35(2), 499-518. https://doi.org/10.6018/rie.35.2.279241

Katewa, E. y Heystek, J. (2019). Instructional and distributed self-leadership for school improvement: experiences of schools in the Kavango Region. Africa Education Review, 16(2), 1-21.

https://doi.org/10.1080/18146627.2016.1267575

Kumpfer, K. L. (1999). Factors and processes contributing to resilience: the resilience framework. En M. D. Glantz y J. L. Johnson (Eds.), Resilience and development: positive life adaptations (pp. 179-224). Kluwer Academic/Plenum Publishers.

Leithwood, K. (2009). ¿Cómo liderar nuestras escuelas? Aportes desde la investigación. Fundación Chile.

Li, L., Hallinger, P. y Walker, A. (2016). Exploring the mediating effects of trust on principal leadership and teacher professional learning in Hong Kong primary schools. Educational Management Administration and Leadership, 44(1), 20-42. https://doi.org/10.1177/1741143214558577

Lin, N., Cook, K. y Burt, R. S. (Eds.). (2008). Social capital theory and research. Aldine de Gruyter.

López, S., Civís, M. y Molina, J. L. (2018). La influencia del capital social en el desarrollo profesional de maestros noveles: una aproximación con métodos mixtos desde el análisis de redes sociales. Profesorado. Revista de Curriculum y Formación del Profesorado, 22(2), 89-110.

https://doi.org/10.30827/profesorado.v22i2.7717

Lumby, J. (2019). Distributed Leadership and bureaucracy. Educational Management Administration \& Leadership, 47(1), 5-19. https://doi.org/10.1177/1741143217711190

Mendoza, I. A., Uribe, J. F. y García, B. R. (2014). Liderazgo y su relación con variables de resultado: un modelo estructural comparativo entre liderazgo transformacional y transaccional en una empresa de entretenimiento en México. Acta de Investigación Psicológica, 4(1), 1412-1429.

Neves, B., Dias de Carvalho, D., Serra, F., Torres, A. y Fraga, S. (2019). Social capital in transition(s) to early adulthood: a longitudinal and mixed-methods approach. Journal of Adolescent Research, 34(1), 85-112. https://doi.org/10.1177/0743558418755685 
Ordóñez, M. L, Botello, B. y Moreno, L. E. (2017). Estilos de liderazgo desde la perspectiva de rango total. Espacio, 38(61), 1-14. https://www.revistaespacios.com/a17v38n61/17386111.html

Osmond-Johnson, P. (2018). Becoming a teacher leader: building social capital through gradual release. Journal of Professional Capital and Community, 4(1), 66-80. https://doi.org/10.1108/jpcc-05-2018-0016

Popp, J., OLáh, J., Kiss, A., Temesi, A., Fogarassy, C. y Lakner, Z. (2018). The socio-economic force field of the creation of short food supply chains in Europe. Journal of Food and Nutrition Research, 58(1), 31-41. http://real.mtak.hu/100392

Porta, L. y Silva, M. (2003). La investigación cualitativa: el análisis de contenido en la investigación educativa. Red Nacional Argentina de Documentación e Información Educativa.

Redondo, G. y Elboj, C. (2018). Liderazgo y creación social: una aproximación a las principales aportaciones. Revista Internacional de Organizaciones, 21, 109-125. https://doi.org/10.17345/rio21.109-125

Rico, C. (2018). Una aplicación de la Teoría de juegos a la mejora en el sector público de los microestados europeos. Revista Enfoques, 16(28), 121-138. https://www.revistaenfoques.cl/index.php/revista-uno/ article/view/484

Rooney, P. K. (2018). A cultural assets model for school effectiveness. Cambridge Journal of Education, 48(4), 445-459. https://doi.org/10.1080/0305764X.2017.1356266

Somprach, K. y Ngang, T. K. (2018). Development of learning leadership Indicators for Thai secondary school principals. Pertanika Journal of Social Science and Humanities, 26(4), 2259-2278.

Tansey, T. N., Bezyak, J., Kaya, C., Ditchman, N. y Catalano, D. (2017). Resilience and quality of life: an investigation of Kumpfer's resilience model with persons with spinal cord injuries. Rehabilitation Counseling Bulletin, 60(3), 163-174. https://doi.org/10.1177/0034355216655146

Trujillo, J. M., López, J. A. y Lorenzo, M. E. (2011). Análisis y descripción de las percepciones del liderazgo resiliente y liderazgo distribuido en torno al ejercicio directivo (2.0) como posibilidad para aprehender y transformar las instituciones educativas. Revista Iberoamericana sobre Calidad, Eficacia y Cambio en Educación, 9(3), 1-29. https://revistas.uam.es/reice/article/view/10206/10308

Ugarte, C. y Naval, C. (2010). Desarrollo de competencias profesionales en la educación superior. Un caso docente concreto. Revista Electrónica de Investigacion Educativa, (Número Especial), 1-14.

https://redie.uabc.mx/redie/article/view/251

Vázquez, S., Liesa, M. y Bernal, J. (2016). El camino hacia la profesionalización de la función directiva: el perfil competencial y la formación del director de centros educativos en España. Perfiles Educativos, 38(151), 158-174. https://doi.org/10.22201/iisue.24486167e.2016.151.54921

Vega, C. y Zavala, G. (2004). Adaptación del cuestionario multifactorial de liderazgo (MLQ forma 5x corta) de B. Bass y B. Avolio, al contexto organizacional chileno (Tesis de Licenciatura). Universidad de Chile.

Vicente de Vera, M. I. y Gabari, M. I. (2019). Liderazgo pedagógico en educación secundaria: aportaciones desde la evaluación de burnout-resiliencia en docentes. International Journal of Educational Leadership and Management, 7(1), 104-134. https://core.ac.uk/download/pdf/228022259.pdf

Weber, M. (2005). Die drei reinen Typen der legitimen Herrschaft [Las tres formas puras de poder legítimo]. Grin.

Weinstein, J. (2017). Liderazgo educativo en la escuela. Nueve miradas. Universidad Diego Portales. 
Yee, N. Y. y Sulaiman, W. S. W. (2017). Resilience as mediator in the relationship between family functioning and depression among adolescents from single parent families. Akademika, 87(1), 111-122.

https://doi.org/10.17576/akad-2017-8701-08

Zheng, W. (2010). A social capital perspective of innovation from individuals to nations: Where is empirical literature directing us? International Journal of Management Reviews, 12(2), 151-183.

https://doi.org/10.1111/j.1468-2370.2008.00247.x 